\title{
Augmentation Grafts in Septorhinoplasty: Our Experience
}

\author{
R. V. Nataraj, Jagade Mohan, Chavan Reshma, Parelkar Kartik, Hanawte Reshma, \\ Singhal Arpita, Kulsange Kiran, Rengaraja Dev, Rao Kartik, Gupta Pallavi \\ Department of Ear, Nose \& Throat and Head and Neck Surgery, Grant Government Medical College, Mumbai, \\ India \\ Email: nataraj.rv@gmail.com
}

Received 8 June 2015; accepted 26 July 2015; published 29 July 2015

Copyright (C) 2015 by authors and Scientific Research Publishing Inc.

This work is licensed under the Creative Commons Attribution International License (CC BY).

http://creativecommons.org/licenses/by/4.0/

(c) (i)

Open Access

\begin{abstract}
Augmentation of nasal tip and/or dorsum forms the keystone of any Septorhinoplasty surgery. The grafts available for augmentation are numerous and varied. Choice of the graft depends upon the type of augmentation required, patient characteristics and, most importantly, the surgeon. In this article, we would like to present our experience with various augmentation grafts. In our experience, autografts are best grafts for augmentation. But in cases of revision surgeries or deficiency of autografts, allografts can be used. Our choice of allograft is Poly Diaxone Sheath or PDS.
\end{abstract}

\section{Keywords}

\section{Septorhinoplasty, Augmentation Grafts, Poly Diaxone Sheath}

\section{Introduction}

Because of its prominent position in the face, many people are self-conscious regarding the shape and size of nose.

Septorhinoplasty is amongst the most frequently performed surgeries in recent times. However this surgery may also be perceived as the most technically demanding of all cosmetic surgeries.

Augmentation in a Septorhinoplasty surgery serves numerous purposes:

1) In case of a flattened dorsum, it helps in raising and straightening the dorsum by augmenting the underlying skeleton-muscular frame work.

2) For projection or definition of nasal tip.

3) For correction of nasal obstruction caused by incompetent nasal valve(s).

4) Volume augmentation.

How to cite this paper: Nataraj, R.V., Mohan, J., Reshma, C., Kartik, P., Reshma, H., Arpita, S., Kiran, K., Dev, R., Kartik, R. and Pallavi, G. (2015) Augmentation Grafts in Septorhinoplasty: Our Experience. International Journal of Otolaryngology and Head \& Neck Surgery, 4, 317-324. http://dx.doi.org/10.4236/ijohns.2015.44054 
An ideal nasal implant, however, does not exist. Although some implant choices exhibit many of the qualities of the ideal implant, no implant satisfies all requirements. The ideal nasal implant should be readily available, inexpensive, inert, nontoxic, non-carcinogenic, sterilizable, easy to sculpt, easily camouflaged, and able to provide volume and mechanical support. Furthermore, the ideal implant should interact favorably with surrounding tissues, maintain its form over time, resist trauma, infection and extrusion, and remain easy to remove [1].

\section{Discussion}

There are various types of implants available. The final choice of the implants depends upon the surgeon. The types of implants can be classified according to the source of origin:

\subsection{Autografts}

These types of grafts are harvested from the same patient and bear closest resemblance to an ideal graft- biocompatible and low rate of rejection \& extrusion, no risk of disease transmission and minimal inflammatory reaction seen. The major limitation with autografts is limited availability and morbidity associated with their harvesting. The types of autografts may include cartilage, bone and soft tissues like fascia and skin.

\subsection{Cartilage}

\section{“THE CARTILAGE IS TO A RHINOPLASTIC SURGEON WHAT WOOD IS TO CARPENTER”.}

Cartilage is most preferred amongst the autografts available as it can provide both support \& volume and can also be easily cut $\&$ molded into desired shape and size. However, the tendency of the cartilage to warp over a period of time remains its greatest drawback.

\subsubsection{Septal Cartilage}

It is the preferred graft for nasal reconstruction as it can be harvested from the same surgical field. However it cannot be used in cases where a large amount of graft is required (Figure 1 and Figure 2).

\subsubsection{Conchal Cartilage}

It is the second most preferred autologus graft. It can be harvested by anterior incision, posterior incision or inferior incision. The two different parts of conchal cartilage used are Concha Cavum and Concha Cymba. Concha Cavum, as the name suggests, is concave in shape and thus finds its use as tip graft or dorsal graft. Whereas Concha Cymba resembles the lateral crura of lower lateral cartilage and therefore is used in cases of deficient or lost (Figure 3 and Figure 4).

\subsubsection{Coastal Cartilage}

It is preferred when abundant cartilage or also a bone is required. This type of graft can be harvested from $5^{\text {th }}$ to $9^{\text {th }}$ ribs, but $7^{\text {th }}$ rib is preferred. Harvesting of coastal cartilage presents with two chief limitations, both of which can be minimized with proper handling techniques. First being the tendency of the cartilage to wrap over time. This drawback can be minimized by separating the cartilage from the perichondrium and carving the cartilage equally from all sides so as to use the central portion of cartilage which has lower tendency to wrap. Second drawback is significant donor site morbidity including pain, incision scar and, in rare cases, pneumothorax. Lastly calcification of the cartilage in elderly may render the graft unsuitable for carving (Figure 5).

\subsubsection{Composite Graft}

A composite graft is a combination of more than one material, typically skin and cartilage with or without bone and fascia. This graft is most commonly harvested from the ear. This graft is used in patients with alar retraction and correct vestibular stenosis.

\section{TYPES OF GRAFTS FOR TIP AND DORSAL AUGMENTATION}

\section{Shield or Sheen graft}

Shield grafts are useful Rhinoplasty surgeries to provide a better tip definition. The name is derived from the shield like shape of the graft. It is placed over the medial crura of the Lower Lateral Lartilages (LLC) extending to the domes or even above (Figure 6). 


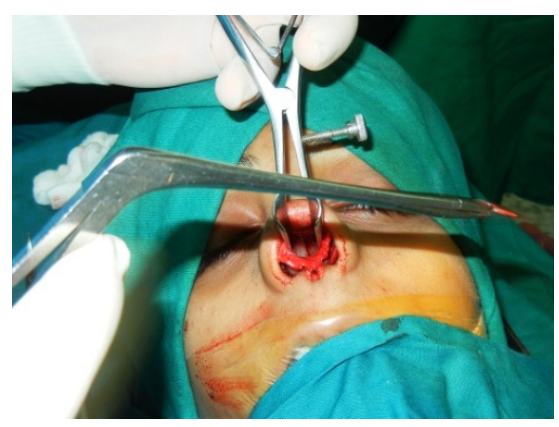

Figure 1. Septal cartilage being harvested.

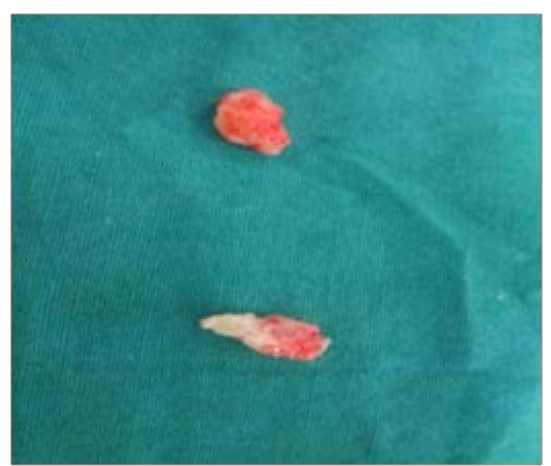

Figure 2. Septal cartilage being harvested.

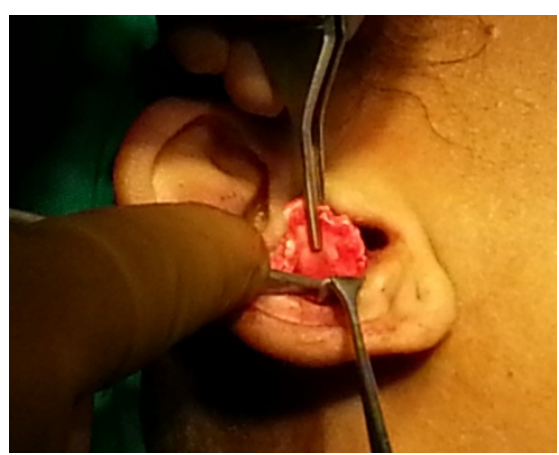

Figure 3. Conchal Cartilage being harvested through anterior approach.

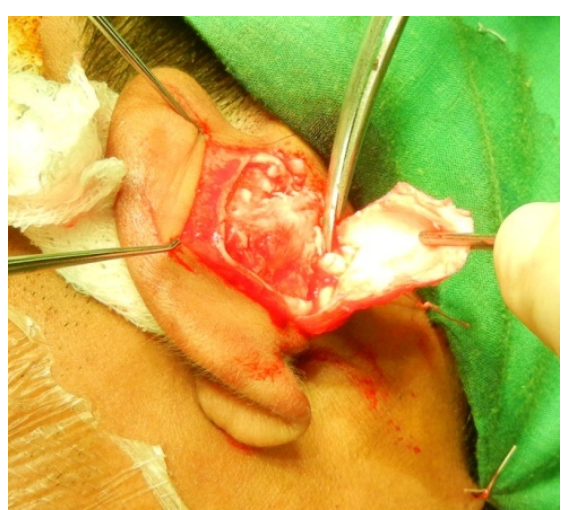

Figure 4. Conchal Cartilage being harvested through anterior approach. 




Figure 5. Harvesting of costal cartilage.

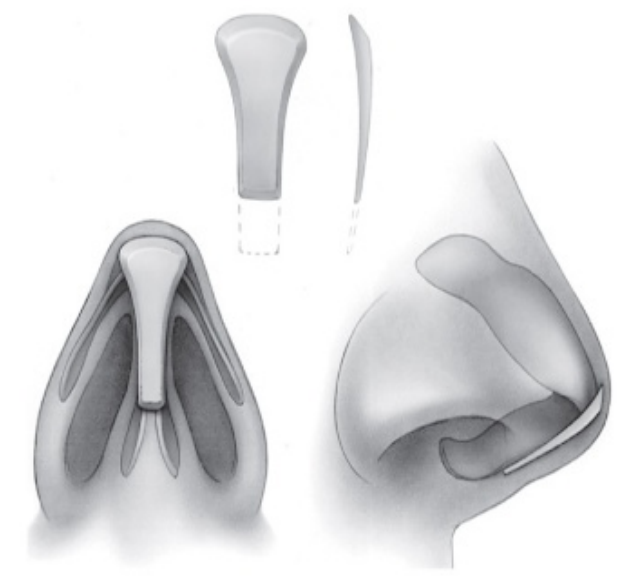

Figure 6. Diagrammatic representation of sheen graft.

Columellar strut graft [2]

Columellar struts are a type of cartilage graft used for tip augmentation in Rhinoplasty. Named so because, in addition to providing better tip definition, it also helps in resizing the columella. It is placed in between the medial crurae of the Lower Lateral Cartilages (LLC) (Figure 7).

Septal extension graft (SEG)

It is a type of cartilage graft which is placed to lengthened the septum and nose. Usually this graft is taken from thick septum, rib cartilage and double layer ear cartilages. It is usually fixed to the nasal septum and provides a better tip definition [3] (Figure 8).

\section{Spreader graft}

Spreader grafts are used to correct the middle vault of the nose and are placed between the upper lateral cartilage and the dorsal septum [4] [5] (Figure 9 and Figure 10).

\section{Umbrella Graft}

These kinds of grafts were first described by Peck in 1989 in his book titled "Techniques in Aesthetic Rhinoplasty”. It mainly consists of a vertical cartilaginous strut placed between the medial crura for better definition and elevation of the tip. It is usually used in conjuncture with a Dorsal Onlay Graft or Dorsal Augmentation Graft [6] (Figure 11).

Dorsal Onlay Graft or Dorsal Augmentation Graft

This is a kind of graft that is used to augment the entire dorsum. It's especially useful in cases of depressed or flattened septum. The most preferred cartilage for carving of a Dorsal Augmentation Graft is rib cartilage [7] [8] (Figure 12).

\section{Sandwich Graft}

In the type of Sandwich Graft, that is used in our institute; an allograft (for example Poly Diaoxone Sheet) is 


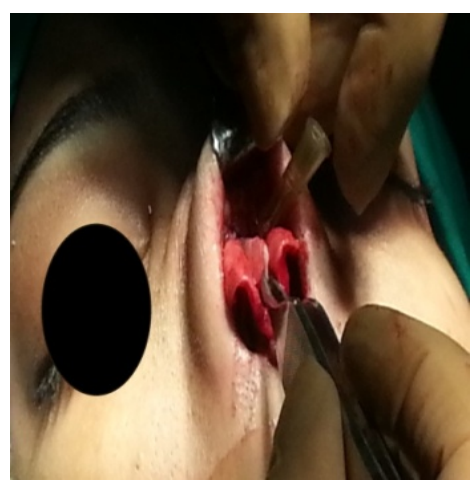

Figure 7. Columellar strut graft being placed.

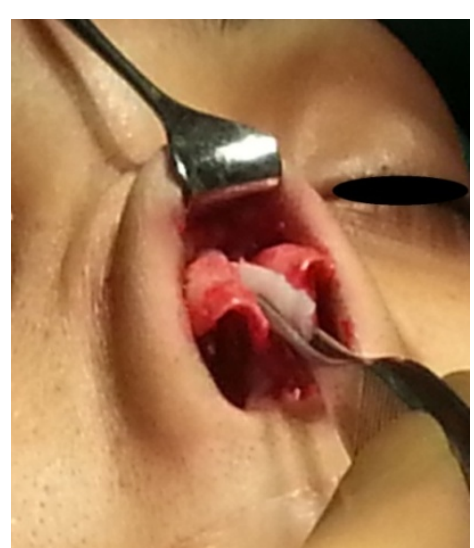

Figure 8. Septal extension graft being placed.

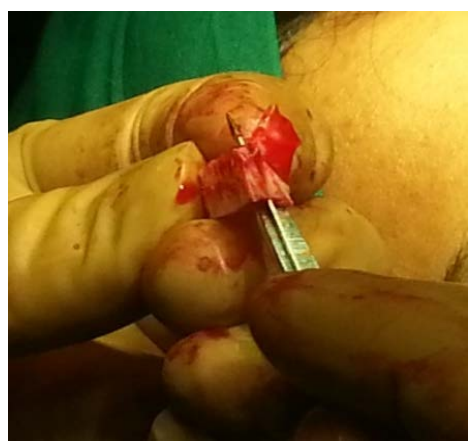

Figure 9 Spreader graft being carved.



Figure 10. Septal extension graft being placed. 


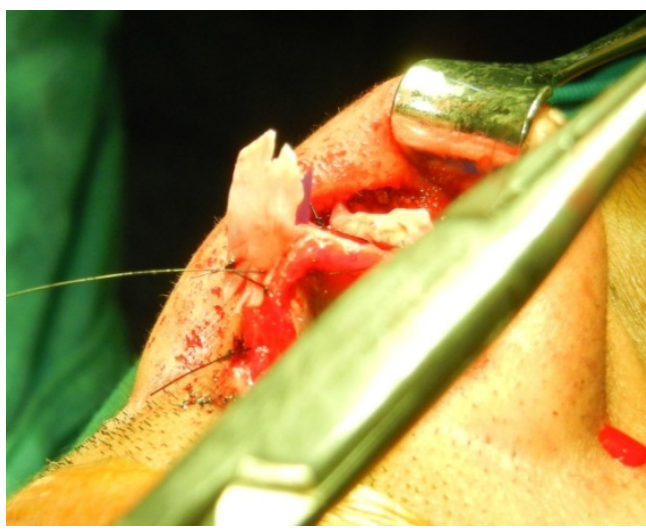

Figure 11. Umbrella graft.

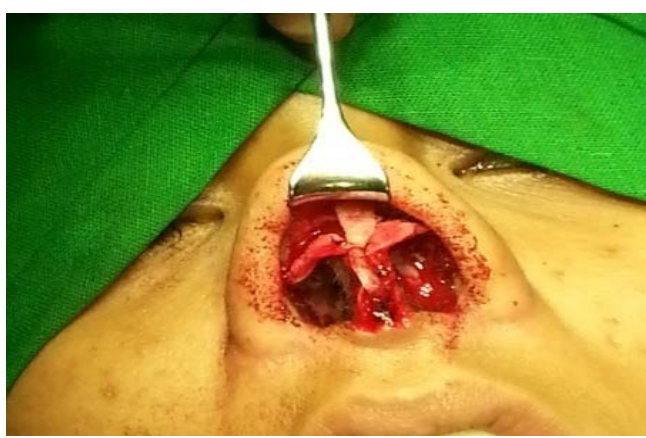

Figure 12. Dorsal augmentation graft.

sandwiched between two autografts like septal cartilage or conchal cartilage. This enables the surgeon to judiciously use the cartilage available at hand in cases of Revision Septorhinoplasty or in cases of cartilage deficiency [7] [8] (Figure 13).

\subsection{Bone}

The most common indication for the use of bone in nose surgery is for dorsal on lay grafting with or without columellar support. Membranous bones (ex-Calvarium) are preferred over endochondral bones (ex-Olecranon process and Iliac crest) because of lower rates of resorption. Chief limitations associated with use of bone as a graft include greater susceptibility to fracture, stiff infrastructure which makes carving difficult and prolonged period of immobilization required for graft fixation [1] [9].

\subsection{Soft Tissue}

Use of soft tissue grafts in dorsal augmentation is very limited because they fail to provide structural support which is vital for dorsal augmentation. Chief use of soft tissue grafts include, camouflaging minor irregularities of skin and adding volume. Most popular amongst the soft tissue grafts is Temporalis fascia graft.

\section{- Homografts}

This kind of graft is derived from soft tissue of a member of the same species. Most commonly used homografts include Irradiated Costal Cartilage (ICC) and Purified human acellular dermal graft [1] [10].

Irradiated Costal Cartilage (ICC)

This type of graft is harvested from the costal cartilage of cadavers and is irradiated with 30,000 to 40,000 Gy of ionizing radiation to decrease the risk of transmission of diseases. The advantages of using ICC graft include low risk of disease transmission, minimal immunogenic reactions and thereby very low rates of extrusion. The chief limitations associated with this kind of graft include tendency to wrap over time and resorption. As described previously for autologous rib cartilage grafting, to decrease the risk of warping, the perichondrium and outer cortex of rib should be removed and the technique of symmetric carving should be employed. 


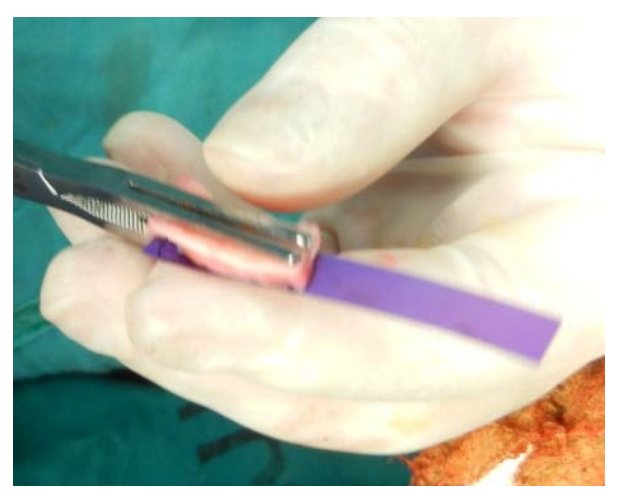

Figure 13. PDS being used to carve sandwhich graft.

\section{Purified Human Acellular Dermal Graft}

The dermis harvested from cadavers is subjected to a process that removes the epidermis and dermal cellular matrix but keeps the extracellular architecture of the dermis intact to facilitate regeneration. The principal uses of this graft are in form of overlay graft, to camouflage over implants, and as volume filling material. Limitations include inability to provide structural support and high rates of resorption.

\section{- Allografts}

These are synthetic grafts which are chemically composed of polymers i.e. repeating units of macromolecules. The unique characteristics required in a nasal implant include pores, malleability and consistency. Pores allow in-growth of the host tissue which decreases the dead space and prevents colonization of bacteria. This also helps in stabilization of the implant [1] [10].

\section{- Silicon}

- Medpore

- Goretex

- PDS

- Fillers

Out of which PDS is most commonly used and fillers are used for touch up.

PDS

PDS is and absorbable implant which is made up of an aliphatic polyester poly-p-dioxanone. PDS is degraded by hydrolysis and is completely absorbed by 25 weeks. It has many unique properties which makes it conducive for nasal surgeries [11] (Figure 14 and Figure 15).

1) Absorbability_PDS plate remains intact during the critical healing process of the first 10 weeks after implantation but is absorbed completely within 25 weeks, leaving no residue and minimal fibrous scar tissue.

2) Structure \& Support-Clinical studies show; PDS Plate provides non-warping support during healing. PDS Plate supports the graft pieces as a guide, bridging and supporting the nasal structure and preventing overlap and bending.

3) Cartilage Management-Patients who have undergone multiple nasal surgeries risk graft depletion. PDS Plate offers a highly versatile and reliable way of making maximal use of many cartilage fragments otherwise discarded, and may reduce the need for secondary cartilage donor site surgery.

4) Versatile - PDS Plate is available in a number of sizes and configurations, one of which is perforated. The product can be trimmed to suit a variety of anatomical conditions and surgical needs including Rhinoplasty and Septoplasty procedures, and the temporary scaffold can be used to construct Columellar Struts, Septal Extension Grafts, Alar Battens, and Upper Lateral Replacement grafts.

\section{Conclusion}

In any nasal reconstructive procedure, the final aesthetic outcome is determined by the graft or implant. An ideal graft does not exist. However Autografts bear closest resemblance to an ideal graft but their availability is limited. In cases of Revision Septorhinoplasty and in cases of limited availability of autografts, allografts play an important role. Amongst all the allograft available at our institute, PDS is the most preferred, as it bears a very 


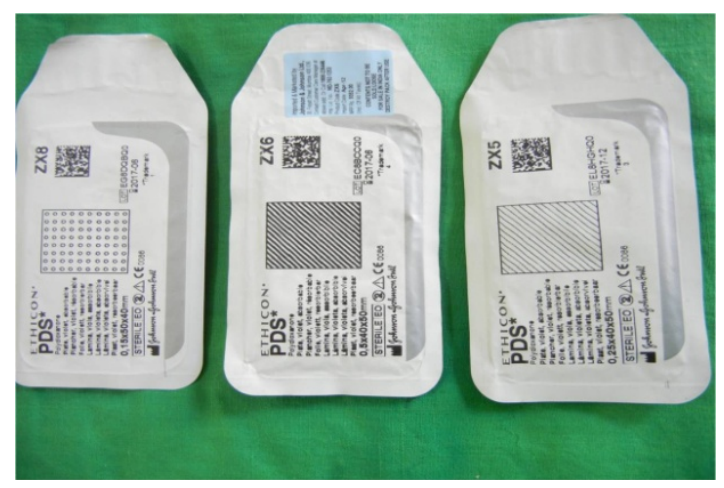

Figure 14. PDS and Introperative use of PDS.

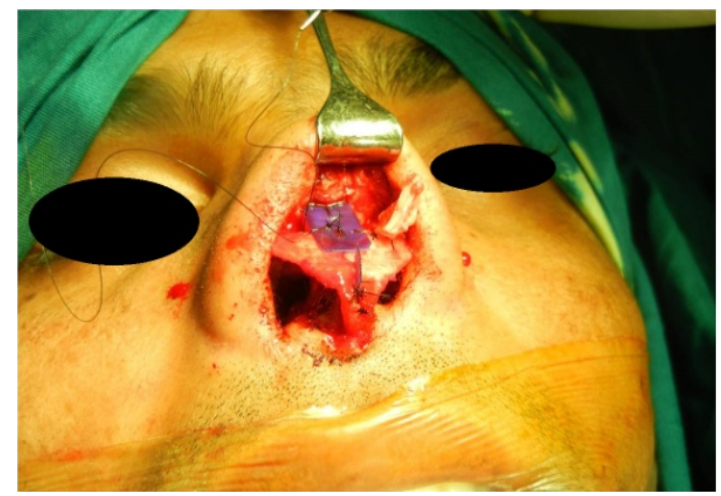

Figure 15. PDS and introperative use of PDS.

close resemblance to an ideal graft.

\section{References}

[1] Romo III, T. and Pearson, J.M. (2008) Nasal Implants. Facial Plastic Surgery Clinics of North America, 16, $123-132$. http://dx.doi.org/10.1016/j.fsc.2007.09.004

[2] Sadeghi, M., Saedi, B., Sazegar, A.A. and Amiri, M. (2009) The Role of Columellar Struts to Gain and Maintain Tip Projection and Rotation: A Randomized Blinded Trial. American Journal of Rhinology and Allergy, 23, e47-e50. http://dx.doi.org/10.2500/ajra.2009.23.3392

[3] Kim, J.H., Song, J.W., Park, S.W., Oh, W.S. and Lee, J.H. (2014) Effective Septal Extension Graft for Asian Rhinoplasty. Archives of Plastic Surgery, 41, 3-11.

[4] Roofe, S.B. (2004) Surgery of the Nasal Valve. Archives of Facial Plastic Surgury, 6, 167-171. http://dx.doi.org/10.1001/archfaci.6.3.167

[5] Teymoortash, A., Fasunla J.A. and Sazgar, A.A. (2012) The Value of Spreader Grafts in Rhinoplasty: A Critical Review. European Archives of Oto-Rhino-Laryngology, 269, 1411-1416. http://dx.doi.org/10.1007/s00405-011-1837-y

[6] Menick, F.J. (1999) Anatomic Reconstruction of the Nasal Tip Cartilage in Secondary and Reconstructive Rhinoplasty. Plastic and Reconstructive Surgery, 104, 2187-2201.

[7] Scattolin, A. and D’Ascanio, L. (2013) Grafts in “Closed” Rhinoplasty. Acta Otorhinolaryngologica Italica, 33, 169-176.

[8] Bussi, M., Palonta, F. and Toma, S. (2013) Grafting in Revision Rhinoplasty. Acta Otorhinolaryngologica Italica, 33, 183-189.

[9] Saeed, M., Hussain, Z. and Mian, F.A. (2012) Augmentation Rhinoplasty with Autologus Iliac Crest Bone Graft. A.P.M.C, 6, 18-21.

[10] Breitbart, A.S. and Ablaza, V.J. (2006) Implant Materials. In: Thorne, C.H., Bartlett, S.P., Beasley, R.W., Aston, S.J., Gurtner, G.C. and Spear, S.L., Eds., Grabb and Smith's Plastic Surgery, 6th Edition, Lippincott Williams \& Wilkins, Philadelphia, Chapter 7, 58-65.

[11] www.pdsflexibleplate.com 